\title{
Modified PTS for PAPR Reduction in Pilot-Assisted Ofdm: Generating New Phase Weighting Factors from the Paley-I Type Hadamard Matrices
}

\author{
Ashish Sachan \\ A.B.E.S. Engineering College, Ghaziabad-201009(U.P.), India
}

\begin{abstract}
High Peak to Average Power Ratio (PAPR) is still a most important challenge in Orthogonal Frequency Division Multiplexing (OFDM) system. This paper proposes a modified Partial Transmit Sequence (PTS) technique which acquires its phase weighting factors from the Paley-I type Hadamard matrices with an objective to reduce the high PAPR in OFDM systems. The proposed technique achieves better PAPR reduction than traditional PTS-OFDM and it also obviate the need for exhaustive search for the viable phase weighting factors in PTS-OFDM, thereby reducing the computational overhead and also avoids the unwanted data rate loss.
\end{abstract}

Index Terms: OFDM, Peak to Average Power Ratio, PTS, Phase Factors

\section{INTRODUCTION}

Orthogonal Frequency Division Multiplexing (OFDM) has been proposed for high data rate in various Wireless Communication standards such as IEEE 802.11, 802.16 and 802.15.3a. The benefits that OFDM brings to wireless communication systems include high bandwidth efficiency, resistance to RF interference, and robustness to multipath fading. However, OFDM signals can exhibit a high peak-to-average power ratio (PAPR), necessitating a power amplifier with a large linear range.

In order to reduce the PAPR of an OFDM signal, many techniques are proposed in the literature: clipping and filtering [1], selected mapping [2], partial transmit sequences [3]. Tone reservation [4], tone injection [4] and Reed-Muller codes [5]. These techniques can be organized into three classes: signal distortion, block coding and signal scrambling.

In this paper, we investigate the pertinent properties of PTS-OFDM with a modification in the traditional technique by selecting the viable phase factors from the Paley-I type Hadamard matrices. Because of the low complexity in implementation of the Hadamard transform matrices and its significance as being the common spreading codes used for MC-CDMA, we investigate the use of these new phase factors in PTS-OFDM system.

The PAPR performances of PTS-OFDM can be well improved with different phase rotation factors. However, the number of available phase rotation factors in PTS determines the size of side information, which causes data rate loss. So in a PTS OFDM system with limited size of side information, it is necessary to pick out the most efficient phase rotation factors to achieve the best PAPR performance. This unwanted data rate loss can be avoided by using the proposed phase sequences because these deterministic phase sequences need not be transmitted along with data and can be generated intact at receiver.

The paper is organized as follows: Section II introduces OFDM signal model by giving the problem statement for PAPR and follows a brief introduction to traditional PTS scheme. Section III provides the elementary idea of Paley-I type Hadamard matrices and generating phase weighting factors for the PTS-OFDM and advancing to the proposed modified PTS-OFDM technique. Section IV evaluates the performance of the proposed technique through simulations. Finally, the paper concludes in Section V by drawing some conclusions.

\section{A. OFDM SYSTEM}

\section{OFDM SYSTEM AND PAPR REDUCTION}

Fig. 1. Shows the block diagram of OFDM system. The base-band modulated symbols are first passed through serial to parallel converter, forming a complex vector of size $\mathrm{N}$.

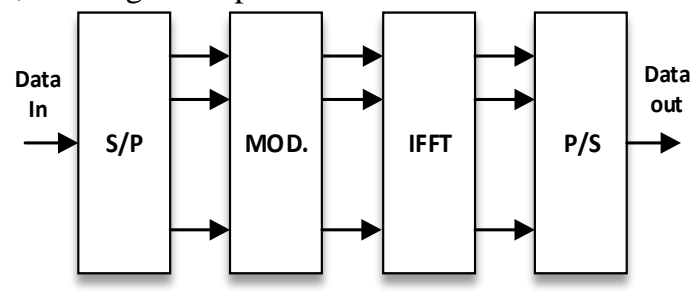

Fig. 1. Basic OFDM System Model 
In an OFDM system, a block of $\mathrm{N}$ symbols is formed as $X=\left[X_{0}, X_{1}, \ldots, X_{N-1}\right]^{\prime}$, and the IFFT transformed the complex baseband representation of OFDM signal consisting of $\mathrm{N}$ subcarriers is given by,

$$
x(\mathrm{t})=\frac{1}{\sqrt{N}} \sum_{n=0}^{N-1} X_{n} \cdot e^{i 2 \pi n \Delta f t}, 0 \leq t \leq N T
$$

Where, $j=\sqrt{-1}, \Delta f$ is subcarrier spacing, and NT is OFDM symbol period.

\section{B. PAPR-PROBLEM STATEMENT}

PAPR is the ratio between the maximum power and the average power of the complex Passband signals [6], that is,

$$
\operatorname{PAPR}\{\tilde{x}(\mathrm{t})\}=\frac{\max \left|\operatorname{Re}\left(\tilde{x}(\mathrm{t}) e^{i 2 \pi f_{c} t}\right)\right|^{2}}{E\left\{\left|\operatorname{Re}\left(\tilde{x}(\mathrm{t}) e^{i 2 \pi f_{c} t}\right)\right|^{2}\right\}}
$$

i.e.

$$
\operatorname{PAPR}\{\tilde{x}(\mathrm{t})\}=\frac{\max |x(\mathrm{t})|^{2}}{E\left\{\left|x(\mathrm{t})^{2}\right|\right\}}
$$

The PAPR definition states that the instantaneous output of an OFDM system often has large fluctuations compared to traditional single-carrier systems. This requires that system devices, such as power amplifiers, $\mathrm{A} / \mathrm{D}$ converters and $\mathrm{D} / \mathrm{A}$ converters, must have large linear dynamic ranges. If this is not satisfied, the peak signal goes into the non-linear region of the device at the transmitter leading to high out of band radiation and intermodulation distortion.

In the literature, the complementary cumulative distribution function (CCDF) is used to evaluate the PAPR reduction performance.

$$
c c d f=\operatorname{Pr}\left(\mathrm{PAPR}>\mathrm{PAPR}_{o}\right)=1-\left(1-e^{-P A P R_{0}}\right)^{N}
$$

\section{PARTIAL TRANSMIT SEQUENCE (PTS)}

A PTS transmitter is shown in Fig. 2. In a typical OFDM system with the PTS approach, the input data block $\mathbf{X}$ is partitioned into $\mathrm{M}$ disjoint sub-blocks or clusters which are combined to minimize the PAP. The data block are represented by the vectors, $\left\{\mathbf{X}^{m}, m=0,1, \ldots, M-1\right\}$ as shown in Fig. 2.

Therefore, we can obtain,

$$
\mathbf{X}=\sum_{m=0}^{M-1} \mathbf{X}^{m}
$$

Where $\mathbf{X}^{m}=\left[X_{0}^{m}, X_{1}^{m}, \ldots, X_{N-1}^{m}\right]$ with, $X_{k}^{m}=X_{k}$, or $0(0 \leq m \leq M-1)$.

Then, the sub blocks $X^{m}$ are transformed into $M$ time-domain partial transmit sequences. Here, it is assumed that the clusters consist of a random set of subcarriers and are of equal size.

$$
\begin{aligned}
\mathbf{X}^{m} & =\left[x_{0}^{m}, x_{1}^{m}, \ldots, x_{L N-1}^{m}\right] \\
& =I F F T_{L N \times N}\left[\mathbf{X}^{m}\right] .
\end{aligned}
$$

These partial sequences are independently rotated by the phase factors, $\mathbf{b}=\left\{b_{m}=e^{i \theta_{m}}, m=0,1, \ldots, M-1\right\}$. These phase factors are weighting factors which are assumed to be pure rotations. The objective is to optimally combine the $M$ sub- blocks to obtain the time-domain OFDM signals with the lowest PAPR. i.e.

$$
\tilde{\mathbf{X}}=\sum_{m=0}^{M-1} b_{m} \mathbf{X}^{m}
$$




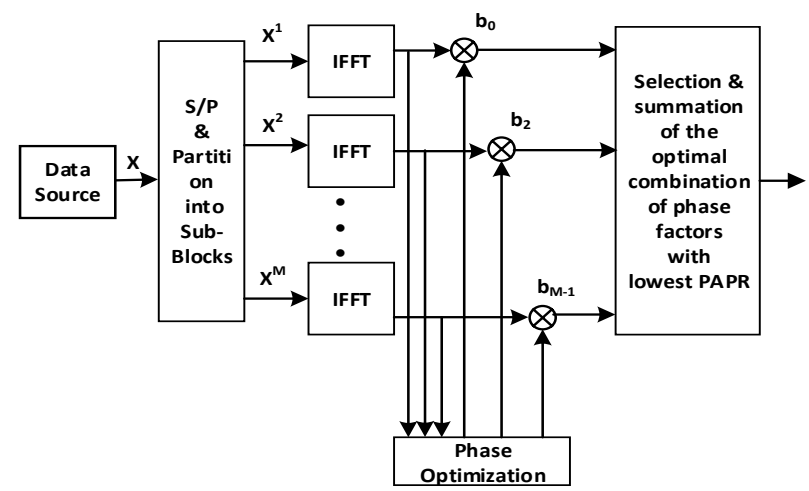

Fig. 2. Block diagram of Partial Transmit Technique (PTS) based OFDM system.

In the PTS approach, the receiver must have knowledge about the generation process of the transmitted OFDM signal. The phase factors must then be transmitted as side information resulting in some loss of efficiency. PTS was first described in [3] as a distortion less PAR method.

\section{A. PALEY-I TYPE HADAMARD MATRICES}

\section{Proposed TEChNiQue}

A major family of Hadamard matrices is the so called Paley Type Hadamard matrices. These families of Hadamard matrices were found by Paley's direct construction using the quadratic residues (that is, the nonzero perfect squares) in a finite field, $G F(q)$ of odd order. In the field, $G F(q)$ half the nonzero elements are quadratic residues of squares and the other half are quadratic non-residues of non-squares.

In particular, +1 is a square and -1 is a non-square if and only if, $q \equiv 3(\bmod 4)$. The version of Paley's constructions given here, and a more accessible proof, may be found in [7].

The quadratic character of $G F(q)$ is the function $\chi$ given by,

$$
\chi(x)= \begin{cases}0 & \text { if } x=0 \\ +1 & \text { if } x \text { is a quadratic residue } \\ -1 & \text { if } x \text { is a quadratic non }- \text { residue }\end{cases}
$$

For $q$ an odd prime power, and an ordering $\left\{g_{0}=0, g_{1}, \ldots, g_{q-1}\right\}$ of $G F(q)$, set $Q=\left[\chi\left(g_{i}-g_{j}\right)\right]$ for $0 \leq i$, and $j \leq q$. Let $S$ be the $(q+1) \times(q+1)$ matrix, then $S=\left(\begin{array}{cc}0 & 1 \\ 1^{T} & Q\end{array}\right)$, where 1 is the all-1s string. Note that $\mathrm{Q}$ is skew-symmetric $\left(Q^{T}=-Q\right)$ when $q \equiv 3(\bmod 4) \&$ symmetric, when $q \equiv 1(\bmod 4)$. Now we can define Paley Type-I Hadamard matrices, $P_{q}$, of order $(q+1)$ as,

$$
P_{q}=\left(\begin{array}{cc}
1 & -1 \\
1^{T} & Q+I_{q}
\end{array}\right)
$$

$$
\begin{aligned}
& \text { 》 H=paleyI (12) } \\
& \mathrm{H}=
\end{aligned}
$$

Fig. 3. An order-12 Paly Type I Hadamard Matrix.

For example, the quadratic residues in $G F(11)$ are 1, 3,4,5,9, and $P_{11}$ of order 12 is, as shown in Fig. 3. The proposed PTS-OFDM technique uses phase weighting factors as derived from the rows of Paley Type-I 
Hadamard matrices and the simulation results obtained are discussed next. Thus $b_{m}$ in Eq. (7) are obtained from the individual row arrays of $P_{q}$.

\section{Simulation ReSUlts}

Extensive simulations in MATLAB $®$ have been performed in order to evaluate the performance of the proposed system as compared with the regular OFDM system as well as those based on the proposed PTSOFDM scheme.

Simulation Parameters for all results are given as in Table I.

TABLE I

SIMULATION PARAMETERS

\begin{tabular}{ll}
\hline \hline \multicolumn{1}{c}{ Parameter } & Value \\
\hline $\begin{array}{l}\text { No. of OFDM Symbol } \\
\text { FFT Size }\end{array}$ & 1000 \\
Oversampling & 64 \\
$\begin{array}{l}\text { Factor (L) } \\
\text { No. of } \\
\text { Subcarriers (N) }\end{array}$ & 4 \\
$\begin{array}{l}\text { Data Tones } \\
\text { Data Tones Index } \\
\text { Modulated Symbols }\end{array}$ & $48+4$ \\
$\begin{array}{l}\text { No. of parallel PTS candidates } \\
\text { (M) }\end{array}$ & 48 QPSK 8 \\
No. of & [-26:-1] [1:26] \\
Phase Factors & B=[ \pm 1$] \quad$ (traditional \\
& PTS) \\
Paley Type-I & Order $4 \& 8$ \\
Hadamard Matrix & \\
$\left(P_{q}\right)$ & \\
\hline \hline
\end{tabular}

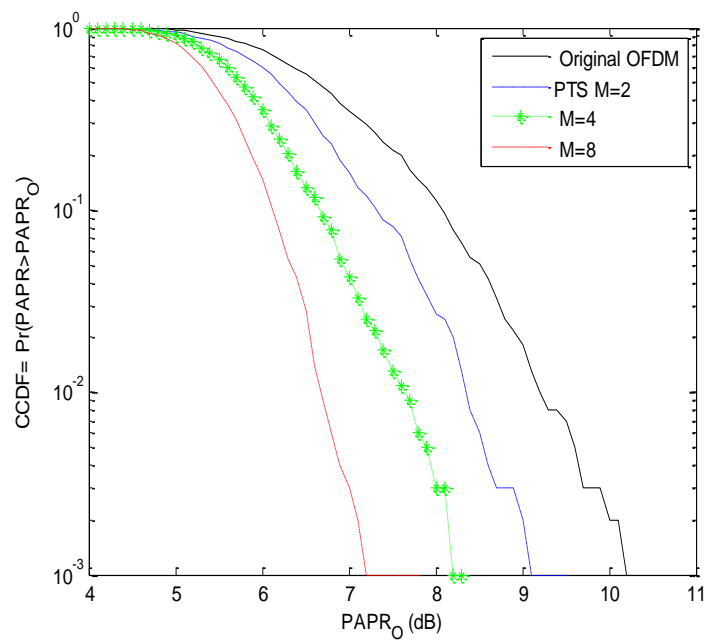

Fig. 4. CCDF of PAPR for the Traditional PTS-OFDM for $M=2,4,8$ (N=64, QPSK, Adjacent PTS Subblocking)

The CCDF (PAPR) for the case of Ordinary OFDM (QPSK, N=64 subcarriers) and traditional PTS-OFDM $(\mathrm{M}=2,4,8)$ is shown in the Fig. 4. It is evident that as M increases PAPR of PTS- OFDM grow better (decreases) though with increased complexity in the system.

Fig. 5 and Fig. 6. , shows the CCDF comparisons of PAPR for Primary OFDM vs. Proposed PTS-OFDM (for 16-QAM symbol mapping and random arrangement of sub-blocks, M). 


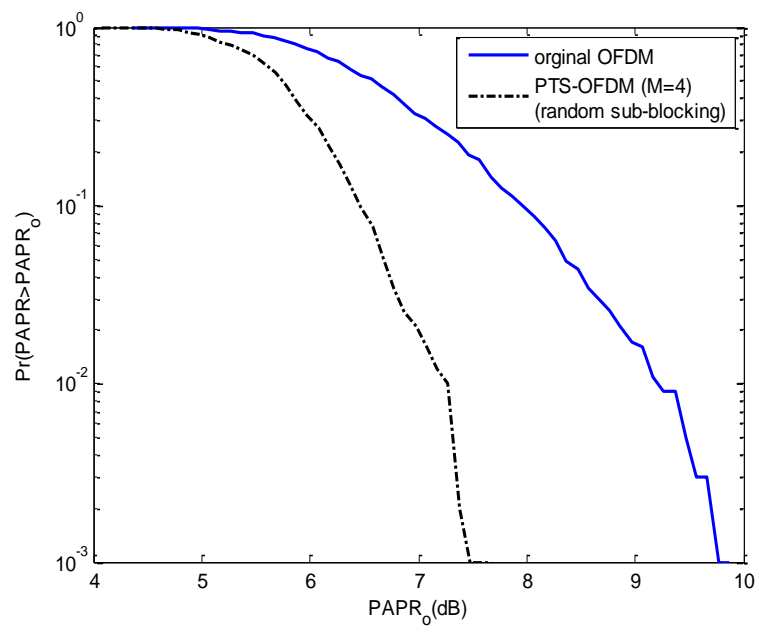

Fig. 5. CCDF of PAPR for Primary OFDM vs. Proposed PTS-OFDM (Random sub-blocking with 16-QAM, $\mathrm{M}=4, \mathrm{~N}=64$ )

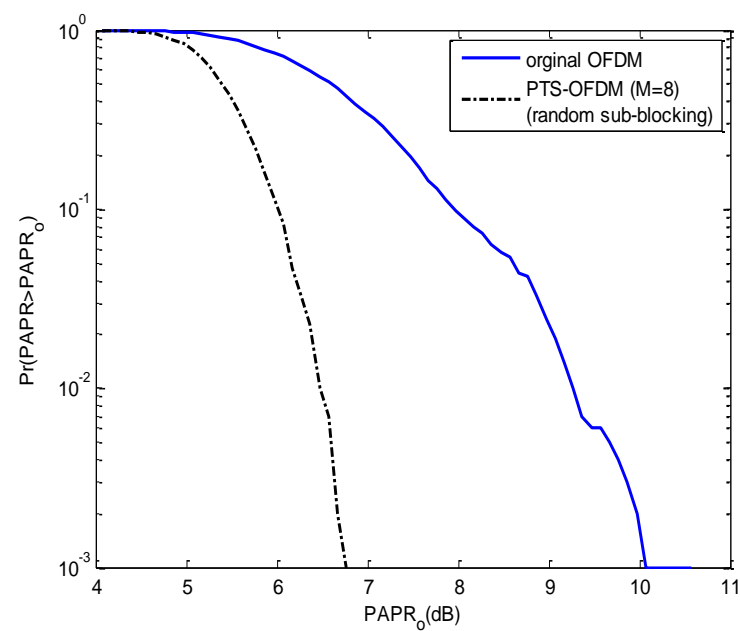

Fig. 6. CCDF of PAPR for Primary OFDM vs. Proposed PTS-OFDM (Random sub-blocking with 16-QAM, $\mathrm{M}=8, \mathrm{~N}=64$ )

Following this, Fig. 7 shows the CCDF comparisons of PAPR for Primary OFDM vs. Proposed PTS-OFDM (for QPSK symbol mapping and random arrangement of sub-blocks, M).

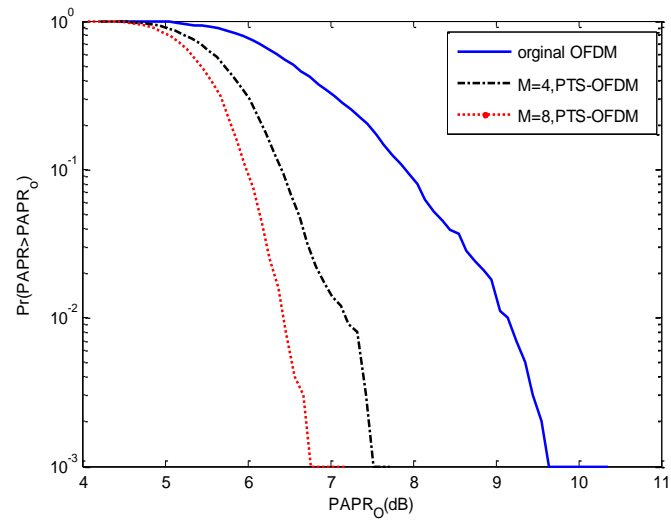

Fig. 7. CCDF of PAPR for Primary OFDM vs. Proposed PTS-OFDM (Random sub-blocking with QPSK, M= $[4,8], \mathrm{N}=64)$.

Also the results of PAPR values for Primary OFDM and Proposed PTS-OFDM along with traditional PTSOFDM, are also summed up in Table II. 
Table II.

[PAPR Comparisons for Primary OFDM and Proposed PTS-OFDM Model]

\begin{tabular}{l|l|l|l}
\hline \hline S/N & $\begin{array}{l}\text { System } \\
\text { Model }\end{array}$ & M & $\begin{array}{l}\text { PAPR } \\
(\mathrm{dB})\end{array}$ \\
\hline (a). & $\begin{array}{l}\text { Primary } \\
\text { OFDM }\end{array}$ & n/a & 10.82 \\
\hline (b). & $\begin{array}{l}\text { Original } \\
\text { PTS-OFDM }\end{array}$ & 4 & 8.2 \\
\cline { 3 - 4 } & $\begin{array}{l}\text { Proposed } \\
\text { PTS-OFDM }\end{array}$ & 4 & 7.2 \\
\cline { 3 - 4 } & & 8 & 6.525 \\
\hline \hline
\end{tabular}

By observing the Table II, we can deduce that w.r.t. Primary OFDM, the proposed PTS-OFDM gives significant PAPR improvement ( $>3 \mathrm{~dB}$, for $\mathrm{M}=4$, and more as $\mathrm{M}$ increases), and in comparison to the traditional PTS-OFDM, though the PAPR improvement is not much but it vastly reduces the computational overhead required in searching the phase weighting factors, and thus making it a better technique.

\section{CONCluSION}

There are two important issues to be solved in the PTS: high computational complexity for searching the optimal phase factors and the overhead of the optimal phase factors as side information need to be transmitted to the receiver for the correct decoding of the transmitted bit sequence, which also incur the inevitable data rate loss. The Paley Type-I Hadamard matrices form one of three main known families of Hadamard matrices which may be constructed directly (at both ends of transmission) from square block designs and thus, they obviate the need for exhaustive search for the viable phase weighting factors in PTS-OFDM, thereby reducing the computational overhead and also avoids the unwanted data rate loss, as well as performs better than traditional PTS-OFDM model in PAPR reduction.

Due to the diversity of PAPR reduction techniques, the subject remains open for further research.

\section{REFERENCES}

[1] Xiaodong Li and Leonard J. Cimini, "Effects of clipping and Filtering on the Performance of OFDM". IEEE, 1997, pp.1634-1638.

[2] R.W. Bauml, et al. , "Reducing the Peak-to-average Power Ratio of Multicarrier Modulation by Selected Mapping". Electronics Letters, 1996,32(22), pp.2056-2057

[3] Muller, S.H. and Huber, J.B. A novel peak power reduction scheme for OFDM. PIMRC'97, vol. 3, pp.1090-1094.

[4] J.Tellado, "Peak to Average Power Reduction for Multicarrier Modulation". Ph.D. dissertation, Stanford University, 2000

[5] J. A. Davis, J. Jedwab, "Peak-to-mean Power Control and Error Correction for OFDM Transmission Using Golay Sequences and ReedMuller Codes". Elect. Lett., vol.33, no. 4, Feb. 1997, pp.267-268.

[6] Palicot, J. and Lou€et, Y. (Sep. 2005) Power ratio definitions and analysis in single carrier modulations. EUSIPCO, Antalya, Turkey.

[7] H. F. Harmuth, Transmission of Information by Orthogonal Functions, $2^{\text {nd }}$ ed., Springer, Berlin, 1972. 